\title{
Comprehensive subcellular topologies of polypeptides in Streptomyces
}

\author{
Konstantinos C. Tsolis ${ }^{1}$, Evridiki-Pandora Tsare ${ }^{2,3}$, Georgia Orfanoudaki ${ }^{4}$, Tobias Busche ${ }^{5}$, Katerina Kanaki ${ }^{4}$, \\ Reshmi Ramakrishnan ${ }^{6}$, Frederic Rousseau ${ }^{6}$, Joost Schymkowitz ${ }^{6}$, Christian Rückert $^{5}$, Jörn Kalinowski ${ }^{5}$, \\ Jozef Anné', Spyridoula Karamanou', Maria I. Klapa ${ }^{2}$ and Anastassios Economou ${ }^{1 *}$ (1)
}

\begin{abstract}
Background: Members of the genus Streptomyces are Gram-positive bacteria that are used as important cell factories to produce secondary metabolites and secrete heterologous proteins. They possess some of the largest bacterial genomes and thus proteomes. Understanding their complex proteomes and metabolic regulation will improve any genetic engineering approach.

Results: Here, we performed a comprehensive annotation of the subcellular localization of the proteome of Streptomyces lividans TK24 and developed the Subcellular Topology of Polypeptides in Streptomyces database (SToPSdb) to make this information widely accessible. We first introduced a uniform, improved nomenclature that re-annotated the names of $\sim 4000$ proteins based on functional and structural information. Then protein localization was assigned de novo using prediction tools and edited by manual curation for 7494 proteins, including information for 183 proteins that resulted from a recent genome re-annotation and are not available in current databases. The $S$. lividans proteome was also linked with those of other model bacterial strains including Streptomyces coelicolor A3(2) and Escherichia coli $\mathrm{K}-12$, based on protein homology, and can be accessed through an open web interface. Finally, experimental data derived from proteomics experiments have been incorporated and provide validation for protein existence or topology for 579 proteins. Proteomics also reveals proteins released from vesicles that bleb off the membrane. All export systems known in S. lividans are also presented and exported proteins assigned export routes, where known.
\end{abstract}

Conclusions: SToPSdb provides an updated and comprehensive protein localization annotation resource for $S$. lividans and other streptomycetes. It forms the basis for future linking to databases containing experimental data of proteomics, genomics and metabolomics studies for this organism.

Keywords: S. lividans TK24, Proteome annotation, Protein subcellular topology, Protein subcellular localization, Database, Secretome, Membranome, Signal peptide, Sortase, Peptidoglycan, Sec system, TAT system

\section{Background}

Members of the genus Streptomyces are soil-dwelling Gram-positive bacteria, belonging to the phylum Actinobacteria with significant industrial and academic interest $[1,2]$. Today, most of the secondary metabolites used in industry and medicine are produced in

\footnotetext{
${ }^{*}$ Correspondence: tassos.economou@kuleuven.be

${ }^{1}$ Laboratory of Molecular Bacteriology, Dpt. of Microbiology and Immunology, Rega Institute, KU Leuven, Herestraat 49, 3000 Leuven, Belgium

Full list of author information is available at the end of the article
}

Streptomyces spp. [3, 4]. In addition, Streptomyces strains have been used as cell factories for heterologous protein expression, including interleukin-6 (IL-6) [5], human mature form of interferon alpha 2 (hIFN-alpha 2) [6], mouse tumor necrosis factor alpha (mTNFalpha) [7], Xeg glucoside hydrolase from Jonesia sp. [8] and a thermophilic cellulase from Rhodothermus [9]. There are two widely used model strains in this family: Streptomyces coelicolor A3(2), used for the production of antibiotics and secondary metabolites [10], and its close relative Streptomyces lividans, which is utilized for heterologous protein production [11]. In our study, 
we focus primarily on S. lividans strain TK24 which has been used for more than 25 years for protein expression and secretion [12-14].

Streptomyces lividans has several advantages that motivate its use as an expression strain. As a Gram-positive bacterium without a mycolic acid layer, the single cell membrane allows protein secretion of folded proteins directly to the culture medium. This avoids accumulation in the periplasm and formation of inclusion bodies seen in E. coli cells. Compared to many other Streptomyces strains, S. lividans TK24 is attractive as a host for genetic manipulation, because it is insensitive to methylated DNA, has a relatively low endogenous protease activity and a collection of vectors and promoters are already available [13]. Streptomyces species export a large amount of their native proteins, including several hydrolases [15], using both the Sec secretion pathway for secretion of nascent, unfolded polypeptides [16] and the TAT pathway for secretion of folded substrates [17] and to a lesser extent the Type VII system [18]. These proteins collectively comprise the "secretome". Another component of the exportome, i.e. the total number of non-cytoplasmic polypeptides, is the membranome that comprises polypeptides with transmembrane regions (TM) that are embedded in the plasma membrane of the cell and many of them are involved in transport and sensing. In addition to native proteins, several recombinant proteins have been produced as secretory proteins [13]. However, several parameters, including growth conditions, affect protein production and perhaps secretion, resulting in variable yields of protein recovery in the spent growth medium $[14,19]$. It becomes apparent that better understanding of how protein secretion is regulated in S. lividans TK24 is essential for the broader use of this strain in protein secretion biotechnology.

Comprehensive protein topological information is not available in the currently available databases specialized for Streptomyces such as StreptomeDB (http://www. pharmaceutical-bioinformatics.org/streptomedb/) that focuses on natural products produced by different Streptomyces species [20] and StrepDB (http://streptomyc es.org.uk/) that includes genome annotation information for multiple Streptomyces species. The main access to the latter database is through custom made scripts and is therefore limited for the wider user community. Topology annotation in Uniprot was the result of automated, non-curated annotation and assigned topologies to only $27 \%$ of the proteome. In addition, LocateP provides and automated subcellular topology prediction tool for Gram-positive bacteria with an accuracy $>90 \%$, which due to automatic scoring algorithms includes misclassified proteins comparing with the individual specialized prediction tools [21].
To provide a generally accessible protein subcellular topology tool, we developed the Subcellular Topology of Polypeptides in Streptomyces (SToPSdb) database, containing information of proteome subcellular topology and protein annotation for TK24. Protein localization was assigned using a combination of bioinformatic tools for protein localization and structural motif prediction, similarity-based alignments (BLAST) and available literature. We used five localization prediction tools and conflicting predictions were edited manually. Protein names were re-annotated providing a more detailed description of protein function. This re-annotation is based on the protein amino acid similarity to known proteins of other Streptomyces species as well as the presence of characteristic structural domains identified through search in the corresponding databases. The content of the SToPSdb database is regularly updated based on experimental and newly published data. The structure of SToPSdb was based on the protein localization database for E. coli, STEPdb that we developed previously [22] and on all the formalisms of Uniprot so that, in effect, information entered in SToPSdb can be easily transferred to Uniprot in future updates. We also expect to link SToPSdb to databases that store experimental data for the multi-omics characterization of S. lividans and its reconstructed metabolic and protein-protein interaction models, towards a comprehensive resource for this bacterium. In a first effort in this direction SToPSdb incorporates topology-related proteomics data currently available.

\section{Methods}

Data collection and assignment of subcellular topologies

The recently re-annotated genome sequence of $S$. lividans strain TK24 was used as reference (Busche et al. in preparation). Identified ORFs were translated into proteins and protein localization was assigned using various prediction tools. Five tools were mainly used: SignalP v4.1 for identification of Sec signal peptides [23], LipoP v1.0 for identification of lipoprotein signal peptides (SpII) [24], PredTAT for identification of TAT and Sec signal peptides [25] Phobius for prediction of signal peptide and TM domains [26], and TMHMM v2.0 for identification of TM domains [27]. The default prediction confidence threshold values of each prediction tool were used.

Conflicts between prediction tools were investigated through manual curation, taking into account the specificity of each tool, known homologues, existing literature, and the putative function of each protein based on common structural domains and functional motifs, as described in the InterPro [28], Pfam [29], and SMART [30] databases. The number of tools used to assign a subcellular protein location is included in the topology 
prediction score; thus, this score ranges from values of $0-5$.

The content of SToPSdb is based on the published proteome of Uniprot and extended with 183 new ORFs that resulted from ongoing transcriptome-based genome annotation (Busche et al. in preparation). The content of SToPSdb and Uniprot is matched using Uniprot's primary protein accession number and the gene name (format SLIV_[0-9]). Part of the annotation available in Uniprot, such as protein existence of structural domains are retrieved from Uniprot and retained in SToPSdb. Additional annotation, including the output of the protein prediction tools, annotation notes, references and experimental evidence are added to each protein and are available through SToPSdb. Annotation for protein function was added upon manual curation, based on the existence of known structural domains for each protein, as it is predicted in the InterPro [28], Pfam [29] and START [30] databases.

\section{Matching of the S. lividans and S. coelicolor proteomes} Comparative genome analyses was performed for genomes of S. lividans TK24 (NCBI accession number: NZ_CP009124) and S. coelicolor A3(2) (NCBI Accession Number: NC_003888) by EDGAR 2.0 [31] to identify orthologs in this genomes. For orthology estimation EDGAR 2.0 uses bidirectional bestBLAST [32] hits with a generic orthology threshold calculated from the similarity statistics of the compared genomes using the BLAST Score Ratio Value approach suggested by Lerat et al. [33].

\section{Database implementation}

SToPSdb is a web-based database. The data is organized and accessed through the mySQL database management system. The web interface of SToPSdb was implemented and generated by PHPMaker, a PHP- and Javascript-based Content Management System [http:// www.hkvstore.com/phpmaker/]. Visual interventions have been implemented with the jQuery library. IM topology graphs are plotted using the JpGraph ObjectOriented chart library (http://jpgraph.net/) and subcellular location distributions under the cell cartoon are drawn using the "Highcharts". The architecture of SToPSdb is identical to the E. coli database, STEPdb, as previously described [22].

SToPSdb can be accessed through an online interface at http://stopsdb.eu with no user restrictions. The user can download all of the provided information via xls files in the separate "Downloads" section.

\section{Results}

\section{Re-annotation of protein description}

A significant limitation and challenge when studying less extensively characterized organisms is the large amount of proteins with generic names/descriptions (e.g. unknown protein, secreted protein etc.). In addition, public databases often use different generic terms without a consistent nomenclature, sometimes different terms are used within the same database. Before proceeding with any protein topology annotation in $S$. lividans, we re-annotated most of these protein names/ descriptions to more specific annotations, based on subcellular topology and structural/functional signatures derived from their homologues and InterPro, Pfam or SMART databases (Additional file 1: Table S1). In many cases this information provides credible predictions for the protein's function in the absence of any experimental evidence. A set of five rules was used to assign protein subcellular localization:

Rule 1: If a protein contains a Sec/TAT or lipoprotein signal peptide and no TM, it is considered a secretory protein of the exportome.

Rule 2: If a protein has at least one predicted TM region (or, optionally, a signal peptide sequence plus at least one TM), it is considered an integral membrane protein of the exportome.

Rule 3: If there is no evidence for TM regions or signal peptides, the protein is defined as cytoplasmic.

Rule 4: If a protein satisfies Rule 3 but experimental or homology data suggests that it can be secreted, then it is considered secreted. This is the case for some piggy-backing TAT substrates and for proteins identified in secreted vesicles (see below).

Rule 5: if a protein is of high similarity with known proteins, then after curation it is assigned a more specific subcellular location (e.g. ribosomal, nucleoid, peripheral inner membrane, peptidoglycan binding).

In addition, manual curation enabled a more insightful annotation about their function and/or subcellular location for several proteins that have so far been assigned temporary generic names. We followed a defined format for protein nomenclature. Names/descriptions are divided into two parts separated by a hyphen, the first describing the subcellular localization group and the second the protein function. For cytoplasmic proteins, no topological suffixes are added. Integral membrane proteins are labeled as "Integral membrane protein -", 
lipoproteins as "Secreted lipoprotein" and extracellular proteins, determined as described below, start with "Secreted protein". For secreted proteins, we include in parenthesis the secretion system that is used for their translocation through the membrane [e.g. (Sec), (TAT) or (T7SS)]. In the second part of the name we include a description of the protein function (e.g. "Esterase"). For example, protein A0A076MHP6 (Uniprot accession) was re-annotated from "uncharacterized protein" to "DNA alkylation repair enzyme" because of its structural similarity with the proteins of this family, as it is described by InterPro, and protein A0A076M7D5 was re-annotated from "secreted protein" to "Secreted protein (Sec)-Solute-binding family 1 protein".

In total, more than 4000 protein descriptions ( $~ 53 \%$ of the proteome) were re-annotated and are included in the database. For example, it has been reported that $S$. coelicolor A3(2) contains two genes encoding Streptomyces subtilisin inhibitor-like (SSI) proteins (genes SCO0762, SCO4010) [34]. For S. lividans TK24, the homologue of the second of these two genes was labeled in Uniprot as "uncharacterized protein", however we assigned the re-annotated protein name based on structural similarity search in InterPro and matching with the homologous proteins of $S$. coelicolor. To help the user, all the re-annotated protein names/descriptions are presented in a downloadable table and online (see below) next to the current Uniprot name and a link to the relevant Uniprot WWW page. Additional representative examples or protein description re-annotation are included in Additional file 2: Table S2.

\section{Annotation of protein sub-cellular topology}

Overall, SToPSdb aims to reduce the number of proteins with unknown topology, based on prediction tools, homologies and/or literature. A defined list of subcellular topologies for each protein is used, following the nomenclature previously presented in STEPdb [22], for the E. coli $\mathrm{K}-12$ proteome annotation (Fig. 1a). The possible topological assignment for each protein corresponds to specific GO (gene ontology) terms. The GO terms are still considered as a main sub-cellular localization classifier for each protein but are less convenient for everyday use. Instead, for simplicity, we use a single letter formalism introduced in EchoLOCATION [35] and refined in STEPSdb [22] (Additional file 3: Table S3, Fig. 1b). For Gram-positive bacteria, the proteome is divided into 9 subcellular locations, a subset of the 13 subcellular locations found in Gram-negative bacteria (Fig. 1a). At the highest level, two basic groups can be identified,

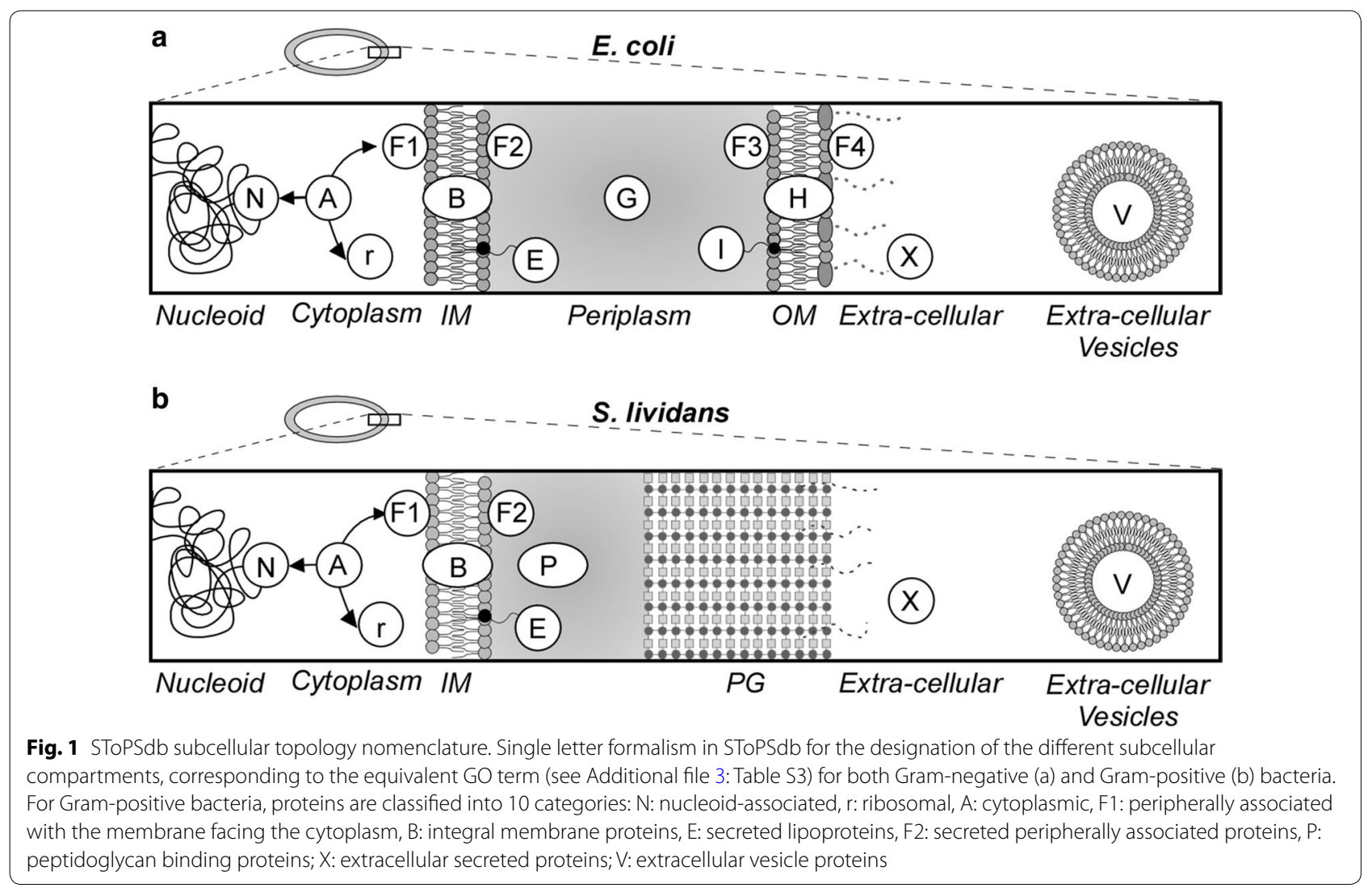


cytoplasmic and exported (exportome) proteins. Cytoplasmic proteins (one letter code: A) are further divided into Nucleoid $(\mathrm{N})$, ribosomal $(\mathrm{r})$, or Peripheral inner membrane proteins facing the cytoplasm (F1). Exported proteins use any of the appropriate secretion systems of the cell (see below) to become either integrated into the membrane (B; membranome) or to become completely translocated across it (secreted proteins; secretome). The secreted proteins are further separated into four classes. Secreted lipoproteins are peripherally anchored to the outer leaflet of the plasma membrane (after their secretion), via lipids covalently bonded to a cysteinyl residue at the $\mathrm{N}$-terminus of the exported protein $(\mathrm{E})$. Secreted peripheral membrane proteins interact with the outer leaflet of the plasma membrane through noncovalent interactions (F2) and have only been studied in some depth in E. coli [36, 37]. Peptidoglycan binding proteins $(\mathrm{P})$ are secreted proteins that interact with the peptidoglycan layer, and extracellular $(\mathrm{X})$ proteins are secreted beyond the peptidoglycan layer to the extracellular matrix. Dual or more topologies are not unusual, referred to as "moonlighting" [38]. Thus, a protein could be cytoplasmic, could bind to DNA and also to the membrane as a peripheral protein, e.g. the transcription regulator CsgD in E. coli [39] or typical cytoplasmic proteins, consistently found in spent growth media of Streptomyces such as TerE (D6EFX5) and SodF1 (D6ECW7) [40, 41]. Proteins with multiple topologies are separated by comma (e.g. A, r) in the relevant topology column of the protein lists in the database and proteins with undefined subcellular location are labeled with the letter " $U$ ".

Initially, we evaluated the subcellular topology annotation available in Uniprot for the TK24 proteome. This was based on the genomic analysis and subsequent automated annotation (Fig. 2, Table 1; last update July 2017). Of the 7322 products of protein-encoding genes identified in the S. lividans proteome, 2153 proteins $(29 \%)$ had been assigned GO (gene ontology) annotations for Cellular Compartment $(\mathrm{CC})$ via automated proteome annotation programs. This number of proteins corresponds to $29 \%$ of the total proteome leaving the remaining $71 \%$ with undefined topologies.

Next, we re-examined de novo the existing protein topology predictions in Uniprot for the $29 \%$ of the proteome using prediction tools and manual curation as described in "Methods" section. Topology conflicts between prediction tools were resolved by manual curation. A common example of topology prediction conflict is the mis-prediction of signal peptides as $\mathrm{N}$-terminal TM domains. To resolve these problems we used SignalP version 4.0 that takes into account additional features including the length of the hydrophobic helix and the presence of a cleavage site [23].

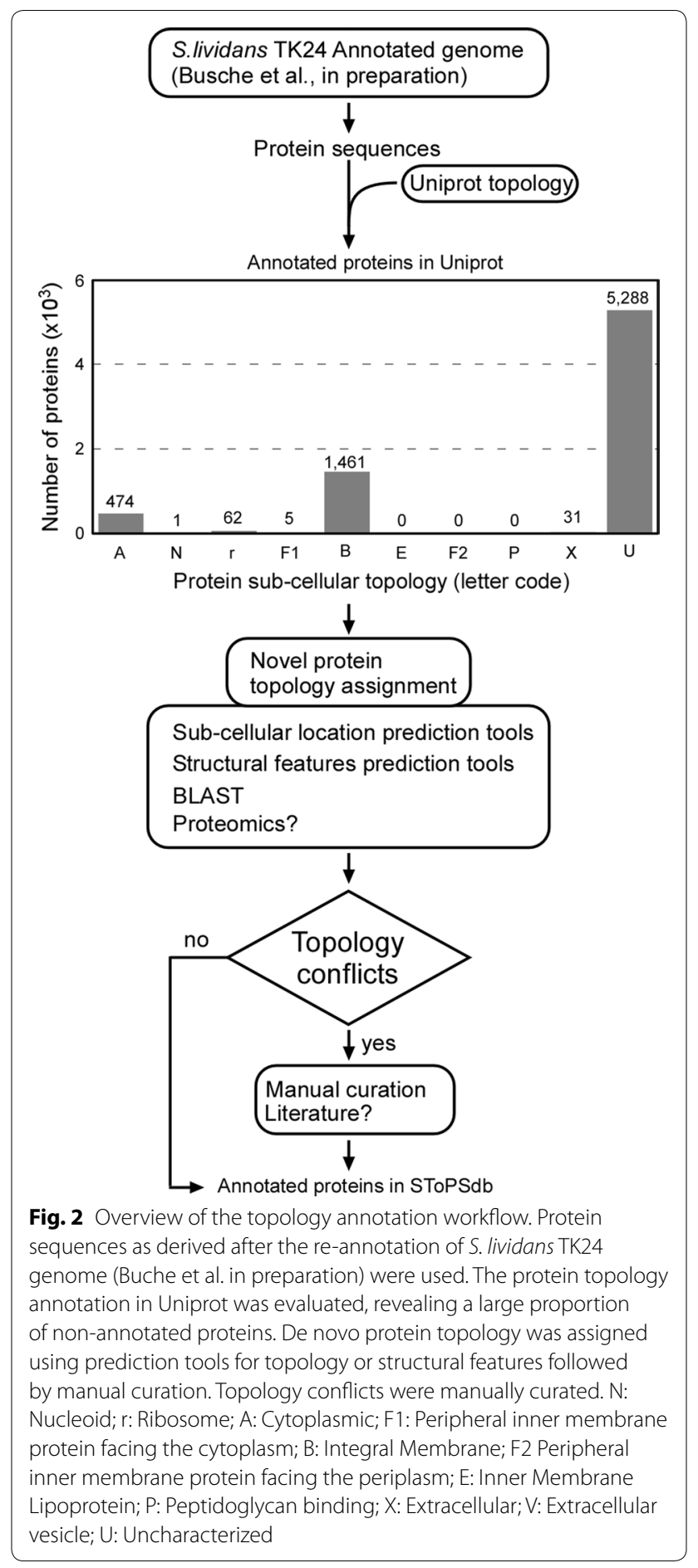

Comparing the protein topology of the current version of Uniprot and SToPSdb, there are topology assignment differences for 12 proteins $(<1 \%$ of the annotated proteins in Uniprot) (Additional file 4: Table S4). 7 of these proteins were annotated as "integral membrane" and 2 of them as "extracellular" in Uniprot and 
Table 1 Summary of previously existing subcellular annotation and comparison with SToPSdb

\begin{tabular}{|c|c|c|c|c|}
\hline & Proteome annotation & Unit of measure & Uniprot & SToPSdb \\
\hline $\begin{array}{l}\text { S. lividans TK24 (total } \\
\text { proteome) }\end{array}$ & & Number of proteins & 7322 & 7505 \\
\hline \multirow{5}{*}{$\begin{array}{l}\text { Subcellular topology } \\
\text { annotations }\end{array}$} & \multirow[t]{2}{*}{ Existing } & Number or proteins & 2153 & 7494 \\
\hline & & $\%$ of reference proteome & $29 \%$ & $>99 \%$ \\
\hline & $\begin{array}{l}\text { Contradicting topology annotations between } \\
\text { Uniprot and SToPSdb }\end{array}$ & Number or proteins & 12 & 12 \\
\hline & \multirow[t]{2}{*}{ Missing/unresolved } & Number or proteins & 5169 & 11 \\
\hline & & $\%$ of reference proteome & $71 \%$ & $<1 \%$ \\
\hline
\end{tabular}

Approximately $30 \%$ of the S. lividans TK24 proteins contains proteome annotation for topology in Uniprot (2153 out of 7322 ). For 12 proteins Uniprot and SToPSdb were not in agreement. Protein topology for this strain was assigned de novo in SToPSdb leaving a small fraction of 11 proteins without topological annotation. Proteins annotated only in one of the two resources are labeled as "Unique (total)"

converted to "cytoplasmic" in SToPSdb, due to lack of evidence of any integral membrane sequence, based on the prediction tools. 3 proteins were described as "integral membrane" in Uniprot and converted to extracellular or secreted lipoprotein in SToPSdb due to the prediction of Sec and Lipoprotein signal peptides. The reason for this discrepancy may be either the automatic protein annotation in Uniprot that may occasionally result in false positive hits, or the wrong prediction of an N-terminal TM domain instead of a signal peptide sequence. We resolved these conflicts by manually evaluating the output of each prediction algorithm used in this study, assigning the corresponding subcellular localization.

Subsequently, we proceeded with the annotation of the $71 \%$ of the proteome that had remained completely un-annotated in Uniprot. The comprehensive re-annotation of the S. lividans TK24 genome and transcriptome (Busche et al. in preparation) added 183 new protein-encoding ORFs, and this updated information has been included in SToPSdb. This brings up the total number of proteins in the S. lividans TK24 proteome to 7505 of which we provide topological annotation for the complete proteome (Table 1).

\section{Proteome-wide analysis of sub-cellular protein topology in S.lividans}

Upon topological annotation of the complete proteome, 2312 proteins (29\% of the total) are found to comprise the exportome and use the Sec, TAT or Type VII secretion pathways either for their insertion into the membrane or their complete translocation across it (Fig. 2). We separate the exportome into the integral membrane proteome or "membranome" (1571 proteins; $21 \%$ of the total proteome) and the secretome (proteins that are completely translocated through the membrane). The secretome (742 proteins; $10 \%$ of the
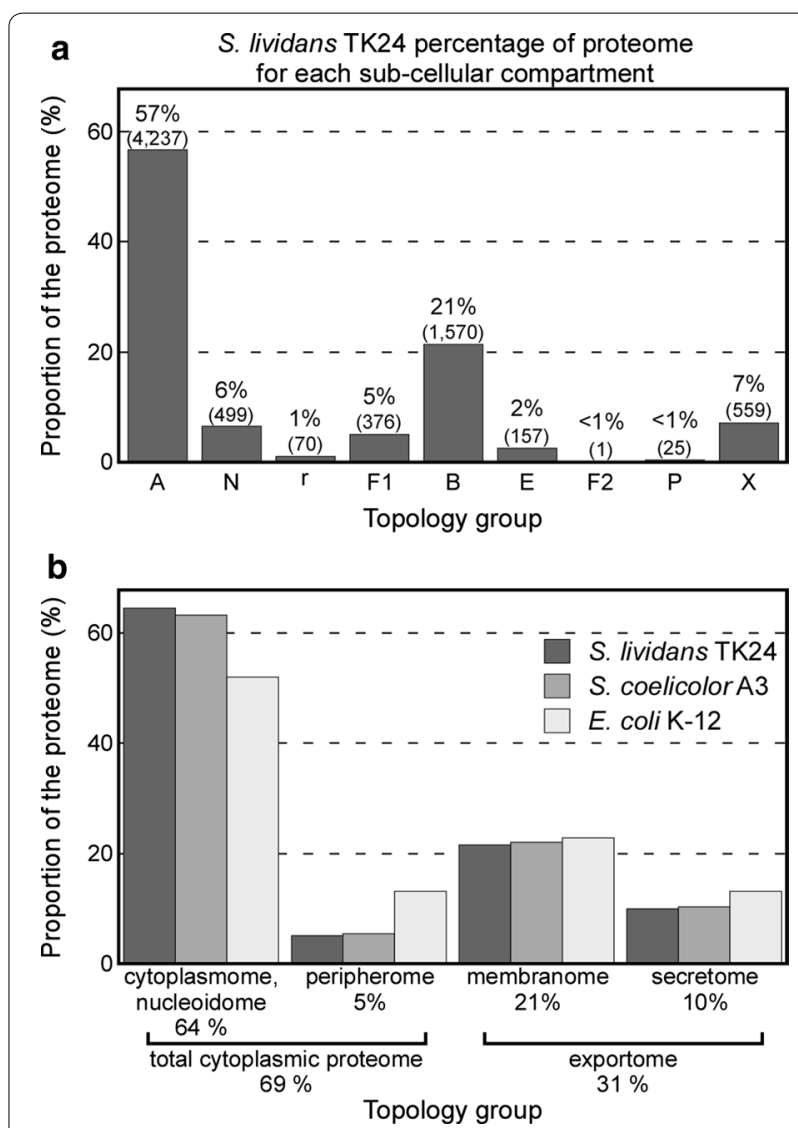

Fig. 3 Distribution of proteins across the subcellular compartments. a Percentage and absolute numbers of proteins corresponding in each subcellular compartment in S. lividans TK24. b Summarized proportion of proteome corresponding in each topology group for the three model bacterial strains S. Iividans TK24, S. coelicolor and E. coli K-12. Topology of the $S$. coelicolor proteome was extrapolated by the S. lividans homologues and additional bioinformatics analyses

total proteome) includes secreted membrane-attached lipoproteins as well as extracellularly secreted proteins that use the Sec (581 proteins, $8 \%$ of total proteome), 
TAT (157 proteins, $2 \%$ of total proteome), or Type VII secretion pathways (3 secreted proteins known to date) (Fig. 3a).

\section{Proteome-wide analysis of protein sub-cellular topology in S. coelicolor}

Having a completely annotated proteome for S. lividans we proceeded to deriving the proteome-wide topology for the closely homologous S. coelicolor strain A3(2), a main model of secondary metabolite production [10]. This was extrapolated based on the respective homologues in $S$. lividans following a proteome-wide BLAST analysis. The two proteomes are very similar and share $94 \%$ average nucleotide identity (ANI) for the matching sequence at the genome level and $93 \%$ of proteins at the protein sequence level, although S. coelicolor has a larger proteome. For the 1064 proteins in S. coelicolor that do not have S. lividans homologues, the localization prediction tools and manual curation were applied as above. Only 2571 out of 8038 proteins of $S$. coelicolor had available subcellular topology annotations in Uniprot. Following our re-annotation process 8029 proteins of S. coelicolor ( $>99 \%$ of the total proteome) have now been assigned subcellular topologies.

Automated prediction of protein sub-cellular localisation for several Gram-positive bacteria is provided by an already established excellent tool-the LocateP database [21]. Upon comparison of protein localization curated in SToPSdb with that provided by LocateP, for S. coelicolor, 374 out of the 8038 proteins annotated in LocateP are in disagreement with SToPSdb and 254 are not directly comparable due to the different labeling of subcellular localization between the two databases (4.7 and 3.2\% respectively) (see Additional file 5: Table S5). Most of the conflicts relate to integral membrane and exported proteins, for which the proportion of proteins with contradictory topology is 5.7 and $21.6 \%$, accordingly. The discrepancy in subcellular localization assignment between the two databases can be attributed to the different type of prediction tools used by each of. To ensure accurate annotation we performed manual curation to each of them, resolving the conflicts between the prediction tools. LocateP has no predictions for the S. lividans proteome.

The generation of complete topological annotations allowed us to compare the two model Streptomyces proteomes with that of the well characterized model bacterium E. coli K-12. Similar proportions of each proteome comprise the cytoplasmic, integral membrane and fully secreted proteins between each of the two Streptomyces strains and E. coli K-12 (Fig. 3b).
In some cases, the same protein has a similar but distinct topology as is the case for FtsY that is an integral membrane protein in Streptomyces [42] but a peripheral protein in E. coli. In other cases, Streptomyces has two homologues of a protein like the peripheral membrane protein SepF [43].

\section{Protein secretion systems}

Many of the components of the Sec secretion machinery of S. lividans TK24 have been annotated in Uniprot (Additional file 6: Table S6). Additional components were identified in SToPSdb, based on structural homology and sequence similarity with characterized proteins (e.g. SecG, YajC and a second homologue of the membrane protein insertase YidC). Single copies of SecA, SecY, $\mathrm{SecE}$, SecG, that form the core essential Sec translocase [16], Trigger factor (TF), signal recognition ribonucleoparticle (SRP; Ffh protein component), and the SRP receptor (FtsY protein) were identified. SecD (A0A076M8T9) and SecF (D6EPR3), that are auxiliary components of the translocase, are present both as separate proteins, and in an additional form in which they are fused in a single polypeptide SecDF (D6EKP9), that was previously reported in S. coelicolor [44, 45]. Functionally, SecD and SecF, and the fused SecDF may function as membrane-integrated chaperones driving using the proton motive force (PMF) [46]. Two copies of the YidC insertase have been identified in S. lividans TK24, D6EMB4 (homologue of O54569 from S. coelicolor) and D6EWK6.

The TAT secretion system secretes folded proteins across the plasma membrane using their own characteristic signal peptides $[17,47] .157$ proteins $(21 \%$ of the predicted TK24 secretome), are expected to be TATsubstrates. 32 of them had been previously experimentally described as TAT substrates in other Streptomyces strains [48-50]. Four proteins of TK24 that carry no predicted TAT signal peptide were additionally annotated as potential TAT-secreted substrates using a "piggy-back" mechanism [22]. Three of them were annotated after being homology matched to the YagG/R/S of E.coli K-12 (Identity $>37 \%$, BlastP E-value $<5 \mathrm{e}-102$ ). The fourth protein, tyrosinase $\mathrm{MelC} 2$, was experimentally shown to be exported in complex with its chaperone MelC1 using the TAT system [50]. Regarding the structural components of the TAT-secretion system, two functional copies of Tat $A$ and one each of TatB and TatC were identified, as seen in S. coelicolor [45, 47] (Additional file 6: Table S6).

The Type VII secretion system characterized in Mycobacterium and other Gram positive bacteria is also present in other Actinobacteria, including Streptomyces [51-53]. Nine T7SS proteins (six structural export machinery components and three secreted proteins) 
were annotated, based on their structural motifs and homologies. Seven of them were mentioned as uncharacterized proteins in Uniprot. Three of these proteins are ESAT-6-like (early secretory antigenic protein) secretory proteins common in $M$. tuberculosis, two are integral membrane serine proteases and four are similar to the structural components EccB, EccC, EccD, and EccE of the mycobacterial T7SS [51] (Additional file 6: Table S6).

Bacteria have evolved poorly understood mechanisms of "non-classical", signal peptide-independent, secretion pathways [54-56]. One such mechanism is using extracellular vesicles [54]. S. lividans produces this type of vesicles that vary in their ultrastructure and macromolecular composition and arise at sites containing peptidoglycan layer defects. They may be related with acquisition of nutrients and virulence against other microorganisms [41]. 26 experimentally detected proteins (12 cytoplasmic, 3 lipoproteins, 11 extracellular secreted proteins) found in such vesicles are annotated in SToPSdb [40, 41].

\section{Mechanisms and properties that influence cell envelope protein topology}

Additional proteins that influence protein subcellular topologies have also been annotated in SToPSdb. Several classes of sortases exist and depending on their class they recognize specific amino acid sequence motifs [57]. Four putative sortases were identified in S. lividans TK24, based on structural motifs, two of them are of class E [58, 59], commonly found in Actinobacteria.

Tail-anchored membrane proteins (TAMPs) [60] have a broad range of functions that are targeted to the membrane post-translationally through C-terminally located TMs sequence $[16,61,62]$. In many cases, no other $\mathrm{N}$-terminal sequences apart from the C-terminal targeting sequences seem to be required and the C-termini alone can localize GFP to the membrane and the $\mathrm{N}$-termini are non-conserved [60]. 73 such proteins have been predicted in the $S$. coelicolor proteome [60]. Of these, 16 have homologues in TK24.

Over-secretion of Sec substrates, could lead to detrimental accumulation of misfolded proteins in the membrane [63]. The CssRS two component system is activated upon secretion stress regulating HtrA-like proteases [64] for misfolded protein degradation [65]. Both the regulatory proteins (CssR, CssS) and the proteases (HtrA1-3, HtrB) have been included in SToPSdb.

Structural motifs can target proteins to the membrane. The conserved bacterial OsmY and nodulation (BON) domain, possibly targets proteins to membranes, through recognition of the phospholipid surface [66]. In S. lividans, 6 previously unannotated BON domain proteins, were annotated as Peripheral plasma membrane proteins facing the cytoplasm.

\section{Web interface and implementation}

The web interface of SToPSdb can be accessed via the URL http://www.stopsdb.eu. On the top-right corner of the webpage there is a search box, allowing quick queries of specific proteins or advanced search options using gene name, Uniprot accession number, or protein name (see also [22]). On the left side of the website is the navigation panel containing three main sub-categories: "Strains", "Proteomics" (see above) and "Downloads and Tools"; a link to the sister database of E. coli STEPdb is also provided. In the Strains panel, the two Streptomyces strains included in SToPSdb are listed; currently these include S. lividans TK24 and S. coelicolor A3(2). By clicking on the "S. lividans TK24" link, a table of proteins will appear on the right, containing the basic identifiers and the protein topology for each protein in this strain. Each protein is linked directly to Uniprot by clicking on the protein accession number. A "more info" button will open a tab with information about the manual curation process (e.g. topology score, references, notes), the prediction tools results, the identified protein family domains, and results of any applied biophysical property prediction tools. Different subsets of the secretory proteins can be selected from the Strains navigation menu. Each of these pages contains a table with the respective proteins and their characterization as described above. In addition, a comparative table between the S. lividans TK24 and $S$. coelicolor protein IDs and a table containing the $S$. coelicolor protein topology, as it is extrapolated from that of S. lividans based on homology, are also included.

The Downloads and Tools menu lists a series of links with supporting information and tools. The "SToPStoGO" page contains the protein topology nomenclature used in the SToPSdb and the corresponding GO terms. "IDMapping" is a tool that reads a list of protein accession numbers returning the corresponding topology. The full content of the database can be found in the "Downloads" page and is available to any user with no restriction. Each protein contains a Uniprot accession number that links SToPSdb with Uniprot, allowing the direct comparison of the SToPSdb entries with the reference proteome database. In addition, the SToPSdb subcellular localization labeling is translated to the corresponding GO terms for cellular compartment, retaining the commonly recognized rules for protein annotation $[67,68]$. Information about terminology, tools and annotation rules used in SToPSdb server are included in the "About" page.

\section{Proteomics data}

The exportome of Streptomyces is of core interest and has attracted a significant effort in SToPSdb. Given the importance of experimentally detected/validated 
proteins in the annotation process, SToPSdb incorporates publicly available experimental data for proteins detected in the exportome ("Proteomics" panel divided in "exportome" and "secretome"). This information can also be downloaded from the "Downloads" page, and the corresponding reference for each study is listed for more detailed datasets. Experimentally detected proteins are listed in one table, and the experimentally detected proteins are marked in the column corresponding to the relevant study. Because the culture medium composition can considerably change the profile of the secreted proteome and has been a focus of many studies $[19,69,70]$, the media used in each study are indicated. Until now experimentally validated TAT substrates (31 proteins) $[48,49]$, proteins detected in extracellular vesicles (26 proteins) [40, 41] or in the exportome of S. lividans TK24 growing in MM-CAS medium (296 proteins) [71], have been presented. An additional comparative proteomics study between $S$. lividans and S. coelicolor growing under low $\mathrm{O}_{2}$ conditions, detected 1832 proteins from which 1486 are cytoplasmic and 346 exported proteins [72].

\section{Discussion}

We developed SToPSdb to provide information on the protein topology annotation of Streptomyces, using as a model organism S. lividans TK24 [7]. SToPSdb is an extension of STEPdb (http://www.stepdb.eu), a database for the comprehensive topological annotation of the Gram-negative model E. coli K-12 [22]. Development of these databases stemmed from the realization that proteomes of critical model microorganisms of biological, biomedical or industrial interest, are still poorly annotated. Moreover, despite the substantial recent progress in automatic annotation, it is obvious that such tools are not yet capable of providing comprehensive and accurate annotations for whole proteomes. This leaves a pressing need for critical manual curation on top of a judicious use of multiple bioinformatics tools. Comprehensive annotation is necessary for all omic-level studies.

An example of the beneficial outcome of such curation is the annotation of proteins involved in the secretory pathway. The YidC insertase, with distinct functions and low sequence identity, has been reported only in some Gram-positive bacteria [73, 74]. The precise function of the second homologue and whether it specializes in the membrane integration of particular substrates remains to be determined. Another group of proteins of potential industrial interest for site-specific protein tagging are the Gram-positive sortases that covalently link secreted proteins with peptidoglycan and control cell shape [75]. Finally, TAMPs in TK24, are tail-anchored membrane proteins that have no predicted Sec or TAT signal peptide and unknown function that awaits future study.
Currently, automated annotation for Streptomyces is provided through the is LocateP database [21]. Comparison of LocateP and SToPSdb for S. coelicolor showed conflicting topological annotation mainly for secreted proteins. The disagreement between these two databases can be attributed to the use of different prediction tools or versions of the same tool. In addition, automated prediction tools will allow a proportion of misclassified proteins, in order to avoid overfitting and generalize well. On the other hand, manual curation can correct more misclassified proteins, but is limited to a small number of organisms that can be annotated.

Emphasis was also given in SToPSdb on the connection of S. lividans TK24 annotation with that of other model strains including $S$. coelicolor and E. coli $\mathrm{K}-12$, based on their homologous proteins. One interesting observation here is that despite the significant phylogenetic differences between Streptomyces and E. coli, their differences in proteome size, transcription regulation diversity and developmental capacities, the percentage of each proteome devoted to cytoplasmic ( $69 \%)$ or exportome $(\sim 31 \%)$ proteins remains rather similar (Fig. 3). The higher percentage of E. coli proteins identified in the peripherome may reflect the dearth of available experimental data in Streptomyces for this biochemically unusual class, rather than a true numerical difference.

We expect SToPSdb to become a reference resource for researchers working with S. lividans, in particular, and streptomycetes in general. We plan to link SToPSdb with integrated omic databases and reconstructed metabolic and protein network models that are currently being developed. This will enhance a comprehensive analysis of the functional processes of S. lividans and can assist in further genetic engineering and biotechnology efforts that can be rationally applied. Towards this direction, we have integrated in this first iteration of the database experimental data derived from proteomics analysis of the exportome. These were derived from multiple proteomics studies that focused on various modes of protein export [41, 48, 49, 71].

\section{Conclusions}

Improvement of the annotation of Streptomyces is an important step towards understanding the complexity of this genus of bacteria and its rational exploitation. SToPSdb provides a proteome annotation database focusing on protein topology. Although we use S. lividans TK24, as the model strain the proteome annotation is linked also to $S$. coelicolor and E. coli $\mathrm{K}-12$, based on homologous proteins. This effort lays the foundation for future developments that will include the 
connection of SToPSdb with other resources containing experimental information at various physiological conditions, providing a reference resource for this organism. SToPSdb can be easily accessed through a web interface at http://stopsdb.eu.

\section{Additional files}

Additional file 1: Table S1. Re-annotated protein names and Identifiers for the proteins included in SToPSdb.

Additional file 2: Table S2. Additional examples of protein description re-annotation in SToPSdb.

Additional file 3: Table S3. Protein topology nomenclature.

Additional file 4: Table S4. Conflicts in protein topology between Uniprot and SToPSdb.

Additional file 5: Table S5. Comparison of SToPSdb and LocateP topological annotation.

Additional file 6: Table S6. Secretion system components in S. lividans TK24.

\section{Abbreviations}

ANI: average nucleotide identity; CC: cellular compartment; ESAT: early secretory antigenic protein; GO: gene ontology; MM-CAS: minimal mediumcasamino acids; PMF: proton motive force; SP: signal peptide; SRP: signal recognition particle; SSI: Streptomyces subtilisin inhibitor; SToPS: Subcellular Topology of Polypeptides in Streptomyces; TAT: twin-arginine translocation; TAMPS: tail-anchored membrane proteins; TM: transmembrane; T7SS: type VII secretion system.

\section{Authors' contributions}

KCT performed topological and functional annotation, bioinformatics analyses and organized the database, EPT and MIK organized and maintain the website and database; $\mathrm{GO}$ and MIK provided bioinformatics analyses and $\mathrm{GO}$ provided the first iteration of the database and the website, KK provided database curation, TB, CR, RR, JK, SK and JA provided annotations; AE conceived, managed and supervised the project, provided manual curation and wrote the paper with contributions from KCT and SK. All authors read and approved the final manuscript.

\section{Author details \\ ${ }^{1}$ Laboratory of Molecular Bacteriology, Dpt. of Microbiology and Immunology, Rega Institute, KU Leuven, Herestraat 49, 3000 Leuven, Belgium. ${ }^{2}$ Metabolic Engineering \& Systems Biology Laboratory, Institute of Chemical Engineering Sciences, Foundation for Research and Technology-Hellas (FORTH/ICE-HT), Patras, Greece. ${ }^{3}$ Department of General Biology, School of Medicine, Univer- sity of Patras, Patras, Greece. ${ }^{4}$ Institute of Molecular Biology and Biotechnol- ogy-FoRTH, P.O. Box 1385, Iraklio, Crete, Greece. ${ }^{5}$ Center for Biotechnology (CeBiTec), Universität Bielefeld, 33594 Bielefeld, Germany. ${ }^{6}$ VIB-KU Leuven Center for Brain \& Disease Research and VIB Switch Laboratory, Department for Cellular and Molecular Medicine, KU Leuven, Herestraat 49, 3000 Leuven, Belgium.}

\section{Acknowledgements}

We thank colleagues of the StrepSynth consortium and the Economou lab for useful discussions. We thank G. Fouskas FORTH/ICE-HT and Y. Kouklinos (FORTH/IMBB) for help with systems administration, creating and maintaining the website.

Database URL: http://stopsdb.eu.

\section{Competing interests}

The authors declare that they have no competing interests.

\section{Availability of data and materials}

All data are available in the SToPSdb website for downloading with no restrictions.

\section{Consent for publication \\ Not applicable.}

\section{Ethics approval and consent to participate}

Not applicable.

\section{Funding}

This work was supported by the European Union project (Grant QLK3CT-2002-02056 to J.K., J.A., M.I.K. and A.E.) and by Grants to A.E. (KU Leuven; KUL-Spa; Onderzoekstoelagen 2013; Bijzonder Onderzoeksfonds); FWO; RiMembR; Vlaanderen Onderzoeksprojecten; \#G0C6814N; EOS project ProFlow \#30550343) and RUN (\#RUN/16/001 KU Leuven; to A.E.). The Switch Laboratory was supported by grants from the European Research Council under the European Union's Horizon 2020 Framework Programme ERC Grant agreement 647458 (MANGO) to JS, R.R. was supported by an Erasmus Mundus fellowship.

\section{Publisher's Note}

Springer Nature remains neutral with regard to jurisdictional claims in published maps and institutional affiliations.

Received: 21 December 2017 Accepted: 8 March 2018

Published online: 15 March 2018

\section{References}

1. Anderson AS, Wellington EM. The taxonomy of Streptomyces and related genera. Int J Syst Evol Microbiol. 2001;51:797-814.

2. Chater KF, Biro S, Lee KJ, Palmer T, Schrempf H. The complex extracellular biology of Streptomyces. FEMS Microbiol Rev. 2010;34:171-98.

3. Craney A, Ahmed S, Nodwell J. Towards a new science of secondary metabolism. J Antibiot (Tokyo). 2013;66:387-400.

4. Rutledge PJ, Challis GL. Discovery of microbial natural products by activation of silent biosynthetic gene clusters. Nat Rev Microbiol. 2015;13:509-23.

5. Zhu Y, Wang L, Du Y, Wang S, Yu T, Hong B. Heterologous expression of human interleukin-6 in Streptomyces lividans TK24 using novel secretory expression vectors. Biotechnol Lett. 2011;33:253-61.

6. Pulido D, Vara JA, Jimenez A. Cloning and expression in biologically active form of the gene for human interferon alpha 2 in Streptomyces lividans. Gene. 1986:45:167-74.

7. Lammertyn E, Van Mellaert L, Schacht S, Dillen C, Sablon E, Van Broekhoven A, Anne J. Evaluation of a novel subtilisin inhibitor gene and mutant derivatives for the expression and secretion of mouse tumor necrosis factor alpha by Streptomyces lividans. Appl Environ Microbiol. 1997;63:1808-13.

8. Sianidis G, Pozidis C, Becker F, Vrancken K, Sjoeholm C, Karamanou S, Takamiya-Wik M, van Mellaert L, Schaefer T, Anne J, Economou A. Functional large-scale production of a novel Jonesia sp. xyloglucanase by heterologous secretion from Streptomyces lividans. J Biotechnol. 2006;121:498-507.

9. Hamed MB, Karamanou S, Olafsdottir S, Basilio JSM, Simoens K, Tsolis KC, Van Mellaert L, Guethmundsdottir EE, Hreggvidsson GO, Anne J, et al. Large-scale production of a thermostable Rhodothermus marinus cellulase by heterologous secretion from Streptomyces lividans. Microb Cell Fact. 2017;16:232

10. Bentley SD, Chater KF, Cerdeno-Tarraga AM, Challis GL, Thomson NR, James KD, Harris DE, Quail MA, Kieser H, Harper D, et al. Complete genome sequence of the model actinomycete Streptomyces coelicolor A3(2). Nature. 2002:417:141-7.

11. Rückert C, Albersmeier A, Busche T, Jaenicke S, Winkler A, Friðjónsson ÓH, Hreggviðsson GÓ, Lambert C, Badcock D, Bernaerts K, et al. Complete genome sequence of Streptomyces lividans TK24. J Biotechnol. 2015;199:21-2. 
12. Anné J, Van Mellaert L. Streptomyces lividans as host for heterologous protein production. FEMS Microbiol Lett. 1993;114:121-8.

13. Anne J, Maldonado B, Van Impe J, Van Mellaert L, Bernaerts K. Recombinant protein production and streptomycetes. J Biotechnol. 2012;158:159-67.

14. Anné J, Economou A, Bernaerts K. Protein secretion in gram-positive bacteria: from multiple pathways to biotechnology. In: Bagnoli F, Rappuoli R, editors. Protein and sugar export and assembly in gram-positive bacteria. Cham: Springer International Publishing; 2017. p. 267-308.

15. Gilbert M, Morosoli R, Shareck F, Kluepfel D. Production and secretion of proteins by streptomycetes. Crit Rev Biotechnol. 1995;15:13-39.

16. Tsirigotaki A, De Geyter J, Sostaric N, Economou A, Karamanou S. Protein export through the bacterial Sec pathway. Nat Rev Microbiol. 2017;15:21-36.

17. Palmer T, Berks BC. The twin-arginine translocation (Tat) protein export pathway. Nat Rev Micro. 2012;10:483-96.

18. Pallen MJ. The ESAT-6/WXG100 superfamily_and a new Gram-positive secretion system? Trends Microbiol. 2002;10:209-12.

19. Anné J, Vrancken K, Van Mellaert L, Van Impe J, Bernaerts K. Protein secretion biotechnology in Gram-positive bacteria with special emphasis on Streptomyces lividans. Biochimica et Biophysica Acta (BBA) Mol Cell Res. 2014;1843:1750-61.

20. Klementz D, Doring K, Lucas X, Telukunta KK, Erxleben A, Deubel D, Erber A, Santillana I, Thomas OS, Bechthold A, Gunther S. StreptomeDB 2.0_an extended resource of natural products produced by streptomycetes. Nucleic Acids Res. 2016;44:D509-14.

21. Zhou M, Boekhorst J, Francke C, Siezen RJ. LocateP: genome-scale subcellular-location predictor for bacterial proteins. BMC Bioinf. 2008;9:173.

22. Orfanoudaki G, Economou A. Proteome-wide subcellular topologies of $E$. coli polypeptides database (STEPdb). Mol Cell Proteom. 2014;13:3674-87.

23. Petersen TN, Brunak S, von Heijne G, Nielsen H. SignalP 4.0: discriminating signal peptides from transmembrane regions. Nat Meth. 2011;8:785-6.

24. Rahman O, Cummings SP, Harrington DJ, Sutcliffe IC. Methods for the bioinformatic identification of bacterial lipoproteins encoded in the genomes of Gram-positive bacteria. World J Microbiol Biotechnol. 2008;24:2377.

25. Bagos PG, Nikolaou EP, Liakopoulos TD, Tsirigos KD. Combined prediction of Tat and Sec signal peptides with hidden Markov models. Bioinformatics. 2010;26:2811-7.

26. Kall L, Krogh A, Sonnhammer EL. A combined transmembrane topology and signal peptide prediction method. J Mol Biol. 2004;338:1027-36.

27. Krogh A, Larsson B, von Heijne G, Sonnhammer EL. Predicting transmembrane protein topology with a hidden Markov model: application to complete genomes. J Mol Biol. 2001;305:567-80.

28. Finn RD, Attwood TK, Babbitt PC, Bateman A, Bork P, Bridge AJ, Chang H-Y, Dosztányi Z, El-Gebali S, Fraser M, et al. InterPro in 2017-beyond protein family and domain annotations. Nucleic Acids Res. 2017;45:D190-9.

29. Finn RD, Coggill P, Eberhardt RY, Eddy SR. The Pfam protein families database: towards a more sustainable future. Nucleic Acids Res. 2016;44:D279-85

30. Letunic I, Doerks T, Bork P. SMART: recent updates, new developments and status in 2015. Nucleic Acids Res. 2015;43:D257-60.

31. Blom J, Kreis J, Spanig S, Juhre T, Bertelli C, Ernst C, Goesmann A. EDGAR 2.0: an enhanced software platform for comparative gene content analyses. Nucleic Acids Res. 2016:44:W22-8.

32. Altschul SF, Gish W, Miller W, Myers EW, Lipman DJ. Basic local alignment search tool. J Mol Biol. 1990;215:403-10.

33. Lerat $E$, Daubin $V$, Moran NA. From gene trees to organismal phylogeny in prokaryotes: the case of the gamma-Proteobacteria. PLoS Biol. 2003;1:E19.

34. Kato JY, Hirano S, Ohnishi Y, Horinouchi S. The Streptomyces subtilisin inhibitor (SSI) gene in Streptomyces coelicolor A3(2). Biosci Biotechnol Biochem. 2005;69:1624-9.

35. Horler RS, Butcher A, Papangelopoulos N, Ashton PD, Thomas GH. EchoLOCATION: an in silico analysis of the subcellular locations of Escherichia coli proteins and comparison with experimentally derived locations. Bioinformatics. 2009;25:163-6.

36. Papanastasiou M, Orfanoudaki G, Koukaki M, Kountourakis N, Sardis MF, Aivaliotis M, Karamanou S, Economou A. The Escherichia coli peripheral inner membrane proteome. Mol Cell Proteom. 2013;12:599-610.

37. Papanastasiou M, Orfanoudaki G, Kountourakis N, Koukaki M, Sardis MF, Aivaliotis M, Tsolis KC, Karamanou S, Economou A. Rapid label-free quantitative analysis of the E. coli BL21(DE3) inner membrane proteome. Proteomics. 2016;16:85-97.

38. Wang G, Xia Y, Cui J, Gu Z, Song Y, Chen YQ, Chen H, Zhang H, Chen W. The roles of moonlighting proteins in bacteria. Curr Issues Mol Biol. 2014;16:15-22.

39. Brombacher E, Baratto A, Dorel C, Landini P. Gene expression regulation by the Curli activator CsgD protein: modulation of cellulose biosynthesis and control of negative determinants for microbial adhesion. J Bacteriol. 2006;188:2027-37.

40. Schrempf H, Koebsch I, Walter S, Engelhardt H, Meschke H. Extracellular Streptomyces vesicles: amphorae for survival and defence. Microb Biotechnol. 2011:4:286-99.

41. Schrempf H, Merling P. Extracellular Streptomyces lividans vesicles: composition, biogenesis and antimicrobial activity. Microb Biotechnol. 2015;8:644-58

42. Shen X, Li S, Du Y, Mao X, Li Y. The N-terminal hydrophobic segment of Streptomyces coelicolor Fts $Y$ forms a transmembrane structure to stabilize its membrane localization. FEMS Microbiol Lett. 2012;327:164-71.

43. Duman R, Ishikawa S, Celik I, Strahl H, Ogasawara N, Troc P, Lowe J, Hamoen LW. Structural and genetic analyses reveal the protein SepF as a new membrane anchor for the $Z$ ring. Proc Natl Acad Sci USA. 2013;110:E4601-10.

44. Zhou Z, Li Y, Sun N, Sun Z, Lv L, Wang Y, Shen L, Li Y-Q. Function and evolution of two forms of SecDF homologs in Streptomyces coelicolor. PLOS ONE. 2014:9:e105237.

45. Zhou Z, Sun N, Wu S, Li YQ, Wang Y. Genomic data mining reveals a rich repertoire of transport proteins in Streptomyces. BMC Genomics. 2016;17:510

46. Tsukazaki T, Mori H, Echizen Y, Ishitani R, Fukai S, Tanaka T, Perederina A Vassylyev DG, Kohno T, Maturana AD, et al. Structure and function of a membrane component SecDF that enhances protein export. Nature. 2011;474:235-8

47. Schaerlaekens K, Van Mellaert L, Lammertyn E, Geukens N, Anne J. The importance of the Tat-dependent protein secretion pathway in Streptomyces as revealed by phenotypic changes in tat deletion mutants and genome analysis. Microbiology. 2004;150:21-31.

48. Widdick DA, Dilks K, Chandra G, Bottrill A, Naldrett M, Pohlschroder $M$, Palmer T. The twin-arginine translocation pathway is a major route of protein export in Streptomyces coelicolor. Proc Natl Acad Sci USA. 2006;103:17927-32.

49. Joshi MV, Mann SG, Antelmann H, Widdick DA, Fyans JK, Chandra G, Hutchings MI, Toth I, Hecker M, Loria R, Palmer T. The twin arginine protein transport pathway exports multiple virulence proteins in the plant pathogen Streptomyces scabies. Mol Microbiol. 2010;77:252-71.

50. Leu WM, Chen LY, Liaw LL, Lee YH. Secretion of the Streptomyces tyrosinase is mediated through its trans-activator protein, MelC1. J Biol Chem. 1992;267:20108-13.

51. Costa TRD, Felisberto-Rodrigues C, Meir A, Prevost MS, Redzej A, Trokter M, Waksman G. Secretion systems in Gram-negative bacteria: structural and mechanistic insights. Nat Rev Microbiol. 2015;13:343-59.

52. Abdallah AM, Gey van Pittius NC, DiGiuseppe Champion PA, Cox J, Luirink J, Vandenbroucke-Grauls CMJE, Appelmelk BJ, Bitter W. Type VII secretion-mycobacteria show the way. Nat Rev Micro. 2007;5:883-91.

53. Fyans JK, Bignell D, Loria R, Toth I, Palmer T. The ESX/type VII secretion system modulates development, but not virulence, of the plant pathogen Streptomyces scabies. Mol Plant Pathol. 2013;14:119-30.

54. Lloubes R, Bernadac A, Houot L, Pommier S. Non classical secretion systems. Res Microbiol. 2013;164:655-63.

55. Bendtsen JD, Kiemer L, Fausbøll A, Brunak S. Non-classical protein secretion in bacteria. BMC Microbiol. 2005:5:58.

56. Nickel W. The mystery of nonclassical protein secretion. A current view on cargo proteins and potential export routes. Eur J Biochem. 2003;270:2109-19.

57. Hendrickx APA, Budzik JM, Oh S-Y, Schneewind O. Architects at the bacterial surface - sortases and the assembly of pili with isopeptide bonds. Nat Rev Microbiol. 2011;9:166-76.

58. Kattke MD, Chan AH, Duong A, Sexton DL, Sawaya MR, Cascio D, Elliot MA, Clubb RT. Crystal structure of the Streptomyces coelicolor sortase E1 transpeptidase provides insight into the binding mode of the novel class E sorting signal. PLOS ONE. 2016;11:e0167763. 
59. Spirig T, Weiner EM, Clubb RT. Sortase enzymes in Gram-positive bacteria. Mol Microbiol. 2011;82:1044-59.

60. Craney A, Tahlan K, Andrews D, Nodwell J. Bacterial transmembrane proteins that lack N-terminal signal sequences. PLOS ONE. 2011;6:e19421.

61. Borgese N, Fasana E. Targeting pathways of C-tail-anchored proteins. Biochim Biophys Acta. 2011;1808:937-46

62. Stroud RM, Walter P. Signal sequence recognition and protein targeting. Curr Opin Struct Biol. 1999:9:754-9.

63. Sarvas M, Harwood CR, Bron S, van Dijl JM. Post-translocational folding of secretory proteins in Gram-positive bacteria. Biochim Biophys Acta. 2004;1694:311-27.

64. Gullon S, Vicente RL, Mellado RP. A novel two-component system involved in secretion stress response in Streptomyces lividans. PLOS ONE. 2012; 7:e48987.

65. Vicente RL, Gullon S, Marin S, Mellado RP. The three Streptomyces lividans $\mathrm{HtrA}$-like proteases involved in the secretion stress response act in a cooperative manner. PLoS ONE. 2016;11:e0168112.

66. Yeats C, Bateman A. The BON domain: a putative membrane-binding domain. Trends Biochem Sci. 2003:28:352-5.

67. Ashburner M, Ball CA, Blake JA, Botstein D, Butler H, Cherry JM, Davis AP, Dolinski K, Dwight SS, Eppig JT, et al. Gene ontology: tool for the unification of biology. Gene Ontology Consortium. Nat Genet. 2000;25:25-9.

68. The Gene Ontology Consortium. Expansion of the Gene Ontology knowledgebase and resources. Nucleic Acids Res. 2017;45:D331-8.
69. Giarrizzo J, Bubis J, Taddei A. Influence of the culture medium composition on the excreted/secreted proteases from Streptomyces violaceoruber. World J Microbiol Biotechnol. 2007;23:553-8.

70. Saadoun I, Al-Omari R, Jaradat Z, Ababneh Q. Influence of culture conditions of Streptomyces sp. (strain S242) on chitinase production. Pol J Microbiol. 2009;58:339-45.

71. Koepff J, Keller M, Tsolis KC, Busche T, Ruckert C, Hamed MB, Anné J, Kalinowski J, Wiechert W, Economou A, Oldiges M. Fast and reliable strain characterization of Streptomyces lividans through micro-scale cultivation. Biotechnol Bioeng. 2017;114:2011-22.

72. Millan-Oropeza A, Henry C, Blein-Nicolas M, Aubert-Frambourg A, Moussa F, Bleton J, Virolle MJ. Quantitative proteomics analysis confirmed oxidative metabolism predominates in Streptomyces coelicolor versus glycolytic metabolism in Streptomyces lividans. J Proteome Res. 2017;16:2597-613.

73. Luirink J, Samuelsson T, de Gier J-W. YidC/Oxa1 p/Alb3: evolutionarily conserved mediators of membrane protein assembly. FEBS Lett. 2001;501:1-5.

74. Funes S, Hasona A, Bauerschmitt H, Grubbauer C, Kauff F, Collins R, Crowley PJ, Palmer SR, Brady LJ, Herrmann JM. Independent gene duplications of the YidC/Oxa/Alb3 family enabled a specialized cotranslational function. Proc Natl Acad Sci USA. 2009;106:6656-61.

75. Popp MW, Antos JM, Grotenbreg GM, Spooner E, Ploegh HL. Sortagging: a versatile method for protein labeling. Nat Chem Biol. 2007;3:707-8.

\section{Submit your next manuscript to BioMed Central and we will help you at every step:}

- We accept pre-submission inquiries

- Our selector tool helps you to find the most relevant journal

- We provide round the clock customer support

- Convenient online submission

- Thorough peer review

- Inclusion in PubMed and all major indexing services

- Maximum visibility for your research

Submit your manuscript at www.biomedcentral.com/submit 